\title{
Does the COVID-19 Pandemic Spell the End for the Direct Ophthalmoscope?
}

\author{
Kendrick C. Shih • Charlene Y. C. Chau • Jonathan C. H. Chan • \\ Jasper K. W. Wong • Jimmy S. M. Lai
}

Received: July 26, 2020 / Published online: August 29, 2020

(C) The Author(s) 2020

\section{ABSTRACT}

Despite advances in ophthalmic diagnostics, the direct ophthalmoscope remains a key clinical skill taught to medical students and is the tool of choice for retina examination among non-ophthalmic physicians. However, in recent years viable alternatives have become available. The coronavirus disease 2019 (COVID-19) pandemic has forced a major re-thinking in medical education worldwide. In this commentary, we examined the current merits and limitations of the direct ophthalmoscope in both the clinical sense and in the context of infection control. Furthermore, we compared the direct ophthalmoscope with alternatives, including commercially available portable non-mydriatic fundus cameras, that would allow a change in focus during ophthalmic teaching. We concluded that the latter will better prepare our medical students for the age of telemedicine and deep-learning systems. While the COVID-19 pandemic will not be the sole reason for the 'death' of the direct ophthalmoscope, the global situation will inevitably serve to expedite

Digital Features To view digital features for this article go to https://doi.org/10.6084/m9.figshare.12816434.

K. C. Shih $(\bowtie) \cdot$ C. Y. C. Chau · J. C. H. Chan ·

J. K. W. Wong · J. S. M. Lai

Department of Ophthalmology, Li Ka Shing Faculty

of Medicine, The University of Hong Kong, Hong

Kong, Hong Kong, SAR

e-mail: kcshih@hku.hk long overdue changes in our teaching of ophthalmic skills to non-ophthalmic physicians.

Keywords: COVID-19; Direct ophthalmoscopy; Medical education; Pandemic

\section{Key Summary Points}

The COVID-19 pandemic necessitates a rethinking of ophthalmic teaching for medical students worldwide.

Direct ophthalmoscopy remains a key clinical skill taught in medical schools worldwide, but for non-ophthalmic physicians it remains a difficult skill to acquire and confidently maintain.

Direct ophthalmoscopy has become even more difficult to teach online in the setting of social distancing. In addition, the skillset itself requires close face-to-face contact with patients, placing examiners at risk of infection.

The availability of non-mydriatic portable fundus cameras allows medical educators a greater emphasis on the interpretation of salient retinal signs of systemic disease. Furthermore, the retina findings can be objectively documented and used in both telemedicine settings and deep-learning systems. 


\section{DIGITAL FEATURES}

This article is published with digital features to facilitate understanding of the article. You can access the digital features on the article's associated Figshare page. To view digital features for this article go to https://doi.org/10.6084/m9. figshare.12816434.

\section{COMMENTARY}

Ocular fundus examination with a direct ophthalmoscope has long been regarded as an essential clinical skill in medical education worldwide. The advantages are its relatively low cost, ease of portability and large $15 \times$ magnified view of the fundus. However, with the widespread use of binocular indirect ophthalmoscopes in specialist ophthalmic practices, the use of the direct ophthalmoscope is mostly limited to non-ophthalmic physicians to identify ophthalmic manifestations of vascular or neurological emergencies in emergency rooms, intensive care units and medical clinics [1]. For diabetic retinopathy screening in community general practices, the use of digital fundus photography since the 1990s, with its much more practical wide $45^{\circ}$ field of view, has mostly supplanted examination by direct ophthalmoscope. Despite direct ophthalmoscopy still being taught in medical schools around the world, it is important to note the steep learning curve associated with its use. Often, when using the direct ophthalmoscope, medical students struggle to achieve a reasonable view of the fundus and thus are much less competent on picking up and interpreting salient signs of neurological or vascular emergencies [2]. Even with the introduction of new educational tools in the last decade, including simulators (Eyesi; VRmagic, Mannheim, Germany), there remains a general lack of confidence in direct ophthalmoscope use among non-ophthalmic physicians worldwide [3].

This article is based on previously conducted studies and does not contain any studies with human participants or animals performed by any of the authors.
The coronavirus disease 2019 (COVID-19) pandemic has posed significant challenges to medical education and clinical care [4]. During this panademic period, we identified two main issues with direct ophthalmoscopy. First, as medical educators, we found it extremely challenging to teach direct ophthalmoscopy to medical students through videoconferencing platforms for a number of reasons, all of which we have outlined the reasons in an earlier publication [5]. In particular, learning to use a direct ophthalmoscope requires a substitute patient to practice on; when mandatory social distancing measures are in place, this is difficult to achieve. Furthermore, and more importantly, the examination is performed with the examiner's own face in close proximity to the patient, requiring the examiner's own cheek to be touching the patient's cheek. Our group has recently reported, using ex vivo experiments, that the human conjunctiva is a potential major gateway for the severe acute respiratory syndrome coronavirus-2 (SARS-CoV-2) to enter the body [6]. This is a significant infection control issue in the context of a highly virulent and transmissible disease. While we do not believe that the pandemic will be the sole reason for the obsolescence of the direct ophthalmoscope, it presents medical educators and clinicians worldwide with the opportunity to identify better long-term solutions for fundus examination by non-ophthalmic physicians.

The recent introduction of affordable cameras for non-mydriatic fundus photography, both portable and fixed, such as the Visuscout 100, a mobile fundus camera produced by Carl Zeiss AG (Oberkochen, Germany), has provided a unique alternative in this panademic climate. Such devices allow for accurate, instant widefield fundus image acquisition by the bedside or in clinics. Rather than spend most of the time and effort on actually finding the fundus, the examiner can be properly trained in fundus photo interpretation of common ophthalmic signs of systemic disease. There are further advantages to this method. Firstly, proper documentation and transfer of fundus findings can be made with the images [7]. Alternatively, the images can be saved for a formal remote evaluation by an ophthalmologist for medico-legal 
reasons. Newer fundus imaging devices (for example, the Optos Silverstone [Optos, Dunfermline, UK]) produce a wide $200^{\circ}$ field of view and come with integrated swept-source optical coherence tomography, thus allowing for stateof-the-art multimodality imaging of the retina and choroid. Furthermore, with the development of deep-learning systems for fundus pattern recognition, the images can be screened by artificial intelligence programs with high accuracy and efficiency. An example of this is the recent validation of an artificial intelligence system in screening for papilledema in ocular fundus photographs [8]. With the COVID-19 pandemic forcing us to re-examine the way we teach ophthalmic skills to medical students, we believe this is one major area that should undergo some long overdue changes that can provide long-lasting benefits to clinical care. In the long run, the pandemic may expedite a shift in focus that will better prepare our medical students to work in an environment where telemedicine and artificial intelligence become increasingly important aspects of healthcare. Despite these changes, it is important to remember that a systematic approach to patient examination will never be obsolete. At all times we need to consider the whole patient when evaluating the back of the eye.

\section{ACKNOWLEDGEMENTS}

Funding. No funding or sponsorship was received for this study or publication of this article. The Rapid Service Fee was funded by the authors.

Authorship. All named authors meet the International Committee of Medical Journal Editors (ICMJE) criteria for authorship for this article, take responsibility for the integrity of the work as a whole, and have given their approval for this version to be published.

Authorship Contributions. Kendrick Co Shih, Charlene Yat-Che Chau, Jonathan CheukHung Chan and Jasper Ka-Wai Wong were involved in writing and editing the manuscript.
Jimmy Shiu-Ming Lai was involved in editing the manuscript and in final approval.

Disclosures. Kendrick Co Shih, Jonathan Cheuk-Hung Chan, Jasper Ka-Wai Wong, Charlene Yat-Che Chau and Jimmy Shiu-Ming Lai have nothing to disclose.

Compliance with Ethics Guidelines. This article is based on previously conducted studies and does not contain any studies with human participants or animals performed by any of the authors.

Open Access. This article is licensed under a Creative Commons Attribution-NonCommercial 4.0 International License, which permits any non-commercial use, sharing, adaptation, distribution and reproduction in any medium or format, as long as you give appropriate credit to the original author(s) and the source, provide a link to the Creative Commons licence, and indicate if changes were made. The images or other third party material in this article are included in the article's Creative Commons licence, unless indicated otherwise in a credit line to the material. If material is not included in the article's Creative Commons licence and your intended use is not permitted by statutory regulation or exceeds the permitted use, you will need to obtain permission directly from the copyright holder. To view a copy of this licence, visit http:// creativecommons.org/licenses/by-nc/4.0/.

\section{REFERENCES}

1. Benbassat J, Polak BC, Javitt JC. Objectives of teaching direct ophthalmoscopy to medical students. Acta Ophthalmol. 2012;90(6):503-7.

2. Mottow-Lippa L. Ophthalmology in the medical school curriculum: reestablishing our value and effecting change. Ophthalmology. 2009;116(7): 1235-6.

3. Kelly LP, Garza PS, Bruce BB, Graubart EB, Newman NJ, Biousse V. Teaching ophthalmoscopy to medical students (the TOTeMS study). Am J Ophthalmol. 2013;156(5):1056-61. 
4. Shih CK, Chan JCH, Lai JSM. Maintenance of ophthalmic specialist out-patient service during the COVID-19 outbreak: The University of Hong Kong experience. Eye (Lond). 2020;34:1241.

5. Shih KC, Chan JC, Chen JY, Lai JS. Ophthalmic clinical skills teaching in the time of COVID-19: a crisis and opportunity. Med Educ. 2020;54:663-4.

6. Hui KP, Cheung M-C, Perera RA, et al. Tropism, replication competence, and innate immune responses of the coronavirus SARS-CoV-2 in human respiratory tract and conjunctiva: an analysis in exvivo and in-vitro cultures. Lancet Respir Med. 2020;8: 687-95.

7. Bidot S, Bruce BB, Newman NJ, Biousse V. Nonmydriatic retinal photography in the evaluation of acute neurologic conditions. Neurol Clin Pract. 2013;3(6): 527-31.

8. Milea D, Najjar RP, Zhubo J, et al. Artificial intelligence to detect papilledema from ocular fundus photographs. N Engl J Med. 2020;382:1687-95. 